\title{
Comparison of Different Microscopic Methods with Conventional TB Culture
}

\author{
Farjana Rahman, ${ }^{1}$ Saurab Kishore Munshi, ${ }^{1}$ S. M. Mostofa Kamal, ${ }^{2}$ A. S. M. Matiur Rahman, ${ }^{3}$ \\ M. Majibur Rahman, ${ }^{1}$ and Rashed Noor ${ }^{1 \%}$ \\ ${ }^{1}$ Department of Microbiology, Stamford University Bangladesh, National Tuberculosis Reference Laboratory, National \\ ${ }^{2}$ Institute of Diseases of the Chest and Hospital, ${ }^{3}$ Bangladesh Academy of Sciences, Dhaka
}

Received 14 February 2011/Accepted 25 April 2011

\begin{abstract}
Tuberculosis is considered as the greatest cause of death worldwide. In the developing countries including Bangladesh, population density, poverty, malnutrition, and highly congested environment may create substantial risk for infection with Mycobacterium tuberculosis. The principal obstacle in the treatment of tuberculosis is the consumption of time and inaccurate diagnosis as well. Therefore, a retrospective study was carried out in order to compare results obtained by both the conventional AFB (Acid Fast Bacilli) microscopy and Lowenstein-Jensen (L-J) culture method for detection of Mycobacterium spp. in clinical samples from different categories of patients. Among one hundred and fifty samples, $83(55.3 \%) \mathrm{AFB}^{+}$ results were found under Bright-Field (BF) microscopy, $78(52 \%) \mathrm{AFB}^{+}$and $91(60.7 \%) \mathrm{AFB}^{+}$results were observed under conventional and Light Emitting Diode (LED) fluorescence microscopy. On L-J culture media, $103(68.7 \%) \mathrm{AFB}^{+}$isolates were found which reveals that the culture could be a gold standard for diagnosis of TB. Significantly, the sensitivity and specificity of LED fluorescence microscopy was higher than those of other microscopic methods compared to culture. The resistance rate of isolates was higher for isoniazid $(65 \%)$ and rifampicin $(63.1 \%)$ than streptomycin $(53.4 \%)$ and ethambutanol $(38.8 \%)$. The isolates showed resistance to more than one drug. Among one hundred and three cases, total $36(24.7 \%)$ cases reported as MDR-TB (resistant to all first-line drugs) in previously treated patients but it was nil in the new cases. Thus, this study emphasizes the need of Directly Observed Treatment Short Course (DOTS) program for selection of proper anti-tubercular drug therapy towards the patient rather than providing them with empirical therapy and thereby reducing the alarming increase of drug resistance.
\end{abstract}

Tuberculosis (abbreviated as TB for tubercle bacillus or Tuberculosis) is a common and often deadly infectious disease caused by mycobacteria in humans, mainly by $M$. tuberculosis (1). Tuberculosis (TB) is a major air- borne infectious bacterial disease. It remains a major worldwide health problem with global mortality ranging from 1.6 to 2.2 million lives per year. The situation is further exacerbated with the increasing incidence of drug resistant TB. Multidrug resistant TB (MDR-TB) includes drug resistance to at least isoniazid (INH) and rifampicin (RIF). After HIV, tuberculosis could be considered as the leading cause of death in the world.

Tuberculosis has been a major public health concern in Bangladesh for long time. The country ranks 6th on the list of 22 highest burdened TB showered countries in the world. It is estimated that 3,00,000 new cases crop up each year, of which about half of them are infectious TB. It is further estimated that about 70,000 people die every year due to TB infection. Hence, each hour eight (2) persons die of the disease for which very effective treatment- Directly Observed Treatment (DOT) with free of cost, is available in Bangladesh. The average TB mortality rate (45 deaths

${ }^{\ddagger}$ Corresponding Author. Mailing address: Dr. Rashed Noor, Dept. of Microbiology, Stamford University Bangladesh, 51, Siddeswari Road, Dhaka, Bangladesh. Phone: +88-02-8354577 (Ext-472), Fax: +88-028363698, Email: noor.rashed@ yahoo.com. per 1,00,000 populations) in Bangladesh is 45 percent higher than that in the Southeast asian region (31 deaths per 1,00,000 population) (3).

At present, diagnosis of TB due to M. tuberculosis (MTB) is most commonly done by using microscopy and culture methods. Most commonly Bright-Field microscope (Zeihl-Neelsen staining) and Fluorescence microscope (Auramine-O staining) are used for the detection of AFB. Most standard laboratory text books and guidelines for laboratories suggest that at least three specimens, preferably collected on three consecutive days, should be submitted to the laboratory for acidfast bacilli (AFB) smear and culture.

In TB endemic areas, most of the cases of TB can be diagnosed correctly by simple and cheap methods which are generally available at peripheral hospital level by AFB microscopy. Although acid fast bacilli (AFB) microscopy and Lowenstein-Jensen (L-J) culture remain the cornerstone of the diagnosis of TB but these traditional bacteriological methods possess several disadvantages. They are either slow or their sensitivity is quite low, especially with clinical samples that contain small number of organisms. This can adversely affect the treatment by either delaying it or by causing inappropriate empiric therapy for $\mathrm{TB}$ to subjects without mycobacterial infections or with atypical mycobacteria (2). Therefore, an urgent requirement of promoting highly sensitive LED fluorescence microscopy led us to design the present study. 


\section{MATERIALS AND METHODS}

Study site and study population. The study was carried out at National Tuberculosis Reference Laboratory (NTRL) of National Institute of Diseases of the Chest and Hospital (NIDCH) from April 2010 to November 2010. Patients of all ages and both sexes who were treated with anti-tubercular drugs but did not respond and patients who did not receive any anti-tubercular drugs were enrolled in the study.

Procedure of sputum collection. One spot of morning sputum sample was collected in a container. On the initial hospital visit, the patient was provided a clean, dry, sterile wide-neck, leak-proof container and requested him or her to cough deeply to produce a sputum specimen.

Microscopic observation. Two direct smear were prepared for staining by Ziehl-Neelsen (Z-N) and Fluorescence (FM) techniques respectively and were examined under microscope following standard operating procedure.

Smear preparation and fixation. For Ziehl-Neelsen staining and auramine-O staining, smears were prepared from yellow purulent portion of the sputum using a sterile bamboo stick. A good smear was spread evenly, 3 $\mathrm{cm}$ by $2 \mathrm{~cm}$ in size in the middle part of the slide which was neither too thick nor too thin. The sputum was left for 15-30 minutes for air drying. The smear was fixed by placing the slide over the hot plate at $85{ }^{\circ} \mathrm{C}$ for about $3-5$ minutes.

Method of conventional bacteriological culture. Lowenstein-Jensen (L-Z) medium is the most widely used matrix for tuberculosis culture. L-J medium containing glycerol favors the growth of $M$. tuberculosis. After decontaminating and concentrating procedure of sputum samples, three or four drops of deposit were inoculated on two slopes of Lowenstein-Jensen media. The media were incubated at $37{ }^{\circ} \mathrm{C}$ after inoculation. They were examined within 3-5 days after inoculation for early recognition of rapidly growing Mycobacterium and of contaminated cultures, followed by examination once a week for at least 45 days. Culture was reported as positive as soon as colonies of characteristic morphology constituted of acid-fast bacilli were recognized. The report of culture was prepared according to the number of colonies.

Identification of $\boldsymbol{M}$. tuberculosis. The final species identification of $M$. tuberculosis was done based on the characteristics such as slow growth, colony morphology, and the typical biochemical features. The identities of the isolates were made by growth rate, colony morphology, $p$-nitrobenzoic acid (PNB) susceptibility, catalase and nitrate reduction tests.

Conventional drug susceptibility test (DST). For performing DST, one loopful colonies was scraped from L-J culture media. A sterile, small thick walled screw-capped glass tube containing 5-7 sterile glass beads and tween 80 solution were taken. The loop was gently shaked over the beads and then mixing was performed. The tube was allowed to stand for 15-30 minutes to settle the large aggregates of bacteria. Another tube containing $4.5 \mathrm{ml}$ tween 80 solution was transferred $(2 \mathrm{ml})$ to the homogenous upper part of the supernatant with similar dimension of the MacFarland standard for the visual comparison with the standard.

Dilution and inoculation of bacterial suspension. Serial dilution of the bacterial suspension was done up to $10^{-5}$. Bacterial suspensions were introduced in drug free medium consisting of growth control (GC) (4). One tube was inoculated with suspension S3 (dilution $10^{-3}$ ) labeled as GC3 and another tube was inoculated with suspension S5 (dilution $10^{-5}$ ), labeled as GC5. Tubes containing the drug isoniazid $(0.2 \mu \mathrm{g} / \mathrm{ml})$, rifampicin $(40 \mu \mathrm{g} / \mathrm{ml})$, streptomycin $(4 \mu \mathrm{g} / \mathrm{ml})$ and ethambutol $(2 \mu \mathrm{g} / \mathrm{ml})$ were inoculated with S3 and S5.

\section{RESULTS}

Among one hundred and fifty patients, in L-J culture method a total of $\mathrm{AFB}^{+}$results were detected in 103 $(43.3 \%)$ cases and $\mathrm{AFB}^{-}$results in $47(31.3 \%)$ cases. In BF microscopy, $83(55.3 \%)$ cases were detected as $\mathrm{AFB}^{+}$results and $67(44.7 \%)$ cases as $\mathrm{AFB}^{-}$. In conventional and LED fluorescence microscopy, $\mathrm{AFB}^{+}$results were detected in $78(52 \%)$ cases and 91 $(60.7 \%)$ cases and negative in $72(48 \%)$ and 59 $(39.3 \%)$ cases.
TABLE 1. Results of L-J culture, BF microscopy, conventional fluorescence microscopy and LED fluorescence microscopy

\begin{tabular}{lccl}
\hline \multirow{2}{*}{$\begin{array}{l}\text { Methods specific } \\
\text { for } \text { M. tuberculosis }\end{array}$} & $\begin{array}{c}\text { Positive } \\
(\%)\end{array}$ & $\begin{array}{c}\text { Negative } \\
(\%)\end{array}$ & P-value \\
\cline { 2 - 3 } & $103(43.3)$ & $47(31.3)$ & $0.000^{\mathrm{S}}$ \\
\hline $\begin{array}{l}\text { L-J culture } \\
\text { BF microscopy } \\
\text { conventional }\end{array}$ & $83(55.3)$ & $67(44.7)$ & $0.083^{\mathrm{NS}}$ \\
$\begin{array}{l}\text { Fluorescence } \\
\text { microscopy }\end{array}$ & $78(52)$ & $72(48)$ & $0.563^{\mathrm{NS}}$ \\
$\begin{array}{l}\text { LED Fluorescence } \\
\text { microscopy }\end{array}$ & $91(60.7)$ & $59(39.3)$ & $0.000^{\mathrm{S}}$ \\
\hline${ }^{\mathrm{S}} \mathrm{Significant}$ & $\mathrm{NS} N{ }^{\mathrm{N}}$ & &
\end{tabular}

${ }^{\text {S }}$ Significant, ${ }^{\text {NS }}$ Not significant

Comparison of results of BF microscopy with culture method to detect $M$. tuberculosis. A total of four cases were $\mathrm{AFB}^{+}$in $\mathrm{BF}$ microscopy, but were negative in L-J culture method among one hundred and fifty patients. In twenty cases, $\mathrm{AFB}^{+}$isolates were observed in L-J culture method which was negative in $\mathrm{BF}$ microscopy. $\mathrm{AFB}^{+}$results were detected in 84 cases for both of the methods and in forty two cases it was negative. These results also indicated that the sensitivity of BF microscopy was $95.6 \%$ and specificity was $91.3 \%$ compared to the L-J culture method.

TABLE 2. Comparison of results of BF microscopy with culture method to detect M. tuberculosis

\begin{tabular}{lccc}
\hline \multirow{2}{*}{ BF microscopy } & \multicolumn{2}{c}{ Culture results } & \multirow{2}{*}{ Total } \\
\cline { 2 - 3 } & Positive & Negative & \\
\hline Positive & 84 & 4 & 88 \\
Negative & 20 & 42 & 62 \\
\hline Total & 104 & 46 & 150 \\
\hline
\end{tabular}

Table 2 was statistically analyzed to predict the sensitivity, specificity, accuracy, Positive Predictive Value (PPV) and Negative Predictive Value (NPV) of BF microscopy against culture method which were $95.6 \%, 91.3 \%, 100 \%, 80.7 \%$ and $67.7 \%$, repectively.

Comparison of results of conventional fluorescence microscopy with culture method to detect M. tuberculosis. Among one hundred and fifty patients, six cases gave $\mathrm{AFB}^{+}$results in conventional fluorescence microscopy, but negative in L-J culture method. In twenty five cases, $\mathrm{AFB}^{+}$isolates were observed in L-J culture method which was negative in the conventional fluorescence microscopy. $\mathrm{AFB}^{+}$ results were detected in seventy nine cases for both of the methods and in forty cases it was found to be negative. These results also indicated that the sensitivity of conventional fluorescence microscopy was $92.9 \%$ and the specificity was $86.9 \%$ compared to those from the L-J culture method. 
TABLE 3. Comparison of results of conventional fluorescence microscopy with culture method to detect M. tuberculosis

\begin{tabular}{lccc}
\hline \multirow{2}{*}{$\begin{array}{l}\text { Conventional } \\
\text { fluorescence } \\
\text { microscopy }\end{array}$} & \multicolumn{2}{c}{ Culture results } & Total \\
\cline { 2 - 3 } & Positive & Negative & \\
\hline Positive & 79 & 6 & 85 \\
Negative & 25 & 40 & 65 \\
\hline Total & 104 & 46 & 150 \\
\hline
\end{tabular}

Table 3 was statistically analyzed to predict the sensitivity, specificity, accuracy, Positive Predictive Value (PPV) and Negative Predictive Value (NPV) of conventional fluorescence microscopy against culture method which were $92.9 \%, 86.9 \%, 100 \%, 92.9 \%$ and $61.5 \%$, respectively.

Comparison of results of LED fluorescence microscopy with culture method to detect $M$. tuberculosis. Among one hundred and fifty patients, two cases were $\mathrm{AFB}^{+}$in LED fluorescence microscopy, but were negative in the L-J culture method. In twelve cases, $\mathrm{AFB}^{+}$isolates were observed in L-J culture method which was negative in LED fluorescence microscopy. $\mathrm{AFB}^{+}$results were detected in 96 cases for both of the methods and in forty cases, it was negative. These results also indicated that the sensitivity of LED fluorescence microscopy was $97.9 \%$ and specificity was $95.2 \%$ compared to L-J culture method.

TABLE 4. Comparison of results of LED fluorescence microscopy with culture method to detect $M$. tuberculosis

\begin{tabular}{lccc}
\hline \multirow{2}{*}{$\begin{array}{l}\text { LED } \\
\text { fluorescence } \\
\text { microscopy }\end{array}$} & \multicolumn{2}{c}{ Culture results } & \multirow{2}{*}{ Total } \\
\cline { 2 - 3 } & Positive & Negative & \\
\hline Positive & 96 & 2 & 98 \\
Negative & 12 & 40 & 52 \\
\hline Total & 108 & 42 & 150 \\
\hline
\end{tabular}

Table 4 was statistically analyzed to predict the sensitivity, specificity, accuracy, Positive Predictive Value (PPV) and Negative Predictive Value (NPV) of LED fluorescence microscopy against culture method which were $97.9 \%, 95.2 \%, 100 \%, 97.9 \%$, and $76.9 \%$, respectively.

Results of DST. Among one hundred three $\mathrm{AFB}^{+}$ isolates, $55(53.4 \%)$ isolates were found to be resistant to streptomycin. The resistance rate for isoniazid and rifampicin was $67(65 \%)$ and $65(63.1 \%)$, respectively. $40(38.8 \%)$ isolates were found to be resistant to ethambutol. This result indicates that the resistance rate was higher in case of isoniazid and rifampicin, which are the most commonly used firstline drugs for the treatment of tuberculosis.

\section{DISCUSSION}

Among communicable diseases, tuberculosis is the second leading cause of death worldwide, killing nearly two million people each year. Most cases are in
TABLE 5. Results of DST

\begin{tabular}{lcccc}
\hline $\begin{array}{l}\text { Antibiotics } \\
(\mu \mathrm{g} / \mathrm{ml})\end{array}$ & Resistant & $\begin{array}{c}\text { Percentage } \\
(\%)\end{array}$ & Sensitive & $\begin{array}{c}\text { Percentage } \\
(\%)\end{array}$ \\
\hline Streptomycin (4) & 55 & 53.4 & 48 & 46.6 \\
Isoniazid (0.2) & 67 & 65 & 36 & 35 \\
Rifampicin (40) & 65 & 63.1 & 38 & 36.8 \\
Ethambutol (2) & 40 & 38.8 & 63 & 61.2 \\
\hline
\end{tabular}
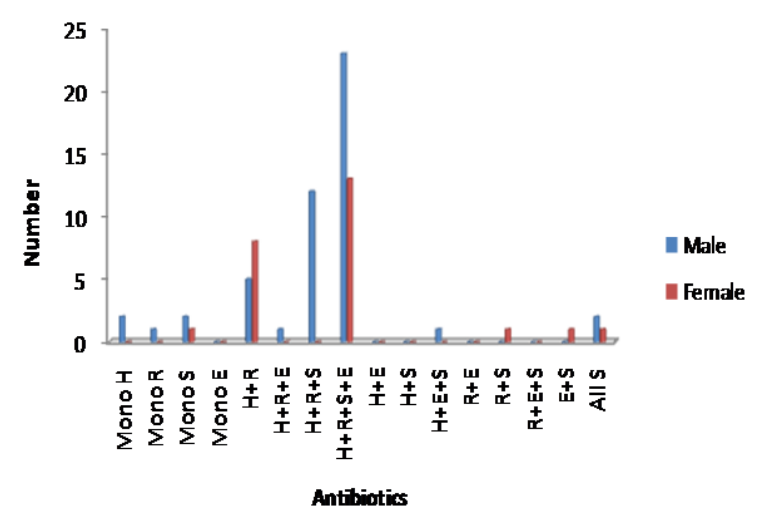

FIG. 1. Indicates the antibiotic resistant pattern of patients (H: Isoniazid, R: Rifampicin, S: Streptomycin, E: Ethambutol). This figure shows that most of the patients were resistant to all of the four first-line anti-tubercular drugs $(\mathrm{H}+\mathrm{R}+\mathrm{S}+\mathrm{E})$. Very few patients were resistant to single drugs (Mono $\mathrm{H}$, Mono $\mathrm{R}$, Mono S, Mono E) and against the combination of two drugs ( $\mathrm{R}+\mathrm{S}, \mathrm{E}+\mathrm{S}, \mathrm{H}+\mathrm{E}, \mathrm{H}+\mathrm{S}, \mathrm{R}+\mathrm{E})$. The resistance rate was also high in case of isoniazid and rifampicin $(\mathrm{H}+\mathrm{R})$ combined.

under developed countries of the world (5). In Bangladesh, Dhaka is a crammed full and grimy area in supplementary precarious to propagate the disease which influenced this study to make the sampling from NIDCH, Mohakhali, Dhaka. The suspected untreated new (primary) patient and previously treated TB patients were included in this study. Random sampling was made from daily incoming suspected patients and TB patients for diagnosis in NIDCH, Mohakhali, Dhaka. According to the clinical demography of the studied patients, this study found that the males $(74 \%)$ were more susceptible to TB than female (26\%). In addition, this study also showed that the incidence among male and female peaked at 21-30 years of age. In this age group, rates among male are usually higher than those among female. The reason of higher male tuberculosis cases than female cases might be explained by the fact that males are actively populated in the community and may come in contact with TB infected persons more frequently. Whereas female members in Bangladesh still reside at home and do not attend at $\mathrm{TB}$ clinic for treatment and therefore the chance of exposure is much less comparatively.

This study suggests to initiate the use of LED fluorescence microscopy in the diagnosis of TB and also helps to sort out the prevalence of MDR-TB patients 
among those who are previously infected as well as medicated with TB drugs.

The results of the present study showed that the LED fluorescence microscopy gave more accurate results compared to $\mathrm{BF}$ (Z-N staining) and conventional fluorescence microscopy, although Z-N stain was considered as the primary procedure for the detection of Mycobacterium spp. Overall seventy nine (52.7\%) AFB positive results were found from AFB microscopic methods, whereas it was negative in forty three $(28.7 \%)$ cases. Lowenstein-Jensen (L-J) medium, which is specific for M. tuberculosis gave one hundred and three (68.7\%) AFB positive results which was better than AFB microscopic results. This result also indicated that the culture method could be a gold standard for diagnosis of TB as stated earlier. L-J media contains malachite green that inhibits the growth of others. The media preparation is comparatively complicated than conventional AFB microscopy and needs efficient staff and good experiment to manipulate the current procedure.

In this study, AFB microscopic methods were compared with culture for diagnosis of TB. In 4 cases positive results were observed in BF microscopy, in 6 cases positive results were observed in conventional fluorescence microscopy and in 2 cases results were positive in LED fluorescence microscopy, but negative on L-J culture media. The reason might be that microscopy sometimes give false positive results and in our condition, it can not distinguish between dead and live bacteria. In such cases, the patients might be treated with anti-tubercular drugs and in the microscopy of these samples, the AFB might be dead. For these reason, the dead isolates did not grow in the L-J culture media. This also reveals that AFB microscopy does not always give accurate results for the diagnosis of TB which made the diagnosis of TB under DOTS comparatively ineffective though it is easy to operate, cheap and quicker rather than culture. The accuracy and frequency of Z-N AFB-microscopy is relatively low compared to other techniques because some Nocardiae share similar type of cell constituent resistant to decolorization with dilute acid and hence give positive result upon microscopic examination. Some non-tuberculous Mycobacterium also shares the same result. This makes difference to diagnose the TB by means of Z-N AFB-microscopy.

In another twenty cases, positive results were found in L-J culture method, which were negative in BF microscopy. In twenty five cases, positive results were observed in L-J culture media but negative in conventional fluorescence microscopy and in twelve cases, results were also positive in L-J culture media but negative in LED fluorescence microscopy. These results indicate that AFB microscopic methods sometimes give false negative results also. The reason might be that the portion of sample which were picked up for staining was not sufficient as high bacterial load (>3000-5000 AFB/ml) is required for detection of AFB. However, most of these can be prevented by restaining the smear.

Another significance of the current work is the statistical analysis on the sensitivity and specificity pattern of AFB microscopic methods. The sensitivity and specificity of BF microscopy was $95.6 \%$ and $91.3 \%$. The sensitivity was $92.9 \%$ and specificity was $86.9 \%$ in conventional fluorescence microscopy. In LED fluorescence microscopy, the sensitivity and specificity was $97.9 \%$ and $95.2 \%$, respectively. Thus, LED fluorescence microscopy had higher sensitivity and specificity than others. In a similar study, Githui et al. (1999) compared the reliability of fluorescence microscopy (FM) and Ziehl-Neelsen (Z-N) staining method for examination of direct smear in the diagnosis of pulmonary tuberculosis. Culture results were used as the gold standard for assessment (6). Specificity was $97 \%$ and $96 \%$ for FM and Z-N methods, respectively. The sensitivity of the FM method was $80 \%$ than that of the Z-N method $65 \%$. So, these studies revealed with the present consistancy study. Fluorescent microscopy offers well-described benefits, compared with conventional light and fluorescence microscopy, for the evaluation of sputum smear samples for tuberculosis and reduces unnecessary labor.

Thus, this study indicates that in the diagnosis of TB, LED fluorescence microscopy had greater sensitivity and specificity than other microscopic methods. In particular, in case of a single specimen, the diagnostic value of culture was quite significant. It is, therefore, possible to conclude that both microscopy and culture can be used for the diagnosis of TB. If only one or two specimens are available, culture method is preferable. The Z-N method has commonly been used around the world, particularly in developing countries, because of its simplicity and low cost (7).

The second part of the study screened the prevalence of drug resistance among patients. Like many developing countries, in Bangladesh, drug resistant tuberculosis is increasing and is a significant threat to tuberculosis control because there are few drugs effective against TB (8). Sporadic survey by Damien Foundation and ICDDR,B shows overall prevalence of MDR $2-5.5 \%$. It was significantly 
higher among persons who previously had received tuberculosis treatment for one month or more; varies from $5.6 \%$ to $15.4 \%$ (9). In this context, DOTS strategy seems to be an answer to control tuberculosis but rapid emergence of drug resistance may arise with a fixed regimen and not with a regimen based on drug susceptibility profile. This occurs because of lack of information about previous history of treatment.

In the present study, DST was performed for IN H, RIF, EMB and SM. The resistant rate was higher in cases of isoniazid and rifampicin than streptomycin and ethambutanol. The present study also can be compared with the work done by Mahadev et al. (2004), where the level of resistance was numerically different (10) and Iqbal et al. (2000) who showed the overall resistance to rifampicin was $26.6 \%$, to isoniazid $23.5 \%$, streptomycin $19.5 \%$, and ethambutol $11.8 \%$ (11).

The emergence of MDR-TB poses a significant threat to TB control activities throughout the world. The complexity of MDR-TB management makes it essential to create new skills to design, plan, implement, and monitor interventions for the management of MDR-TB. More surveillance and immediate therapeutic interventions should be performed in order to combat the threat of MDR-TB to the general population. At present the diagnosis of tuberculosis in Bangladesh is based on AFB (acid fast bacilli) microscopy using Z-N staining. Though it is cheaper and faster, its sensitivity and specificity is less. Therefore, it is necessary to initiate the use of LED fluorescence microscopy for more accurate and rapid detection. The conventional cultural diagnosis is considered as gold standard for diagnosis of TB but is inappropriate for immediate treatment of tuberculosis. These conditions reflect the need of new diagnostic procedure especially molecular methods for detection of tuberculosis in our country as it requires only two days where conventional culture diagnosis takes morc than four weeks. Proper selection and use of antitubercular drugs for treatment of patients is also necessary to reduce the incidence of MDR-TB.

\section{ACKNOWLEDGEMENT}

We thank NTRL Laboratory for providing us with the facilities for all the experiments described here.

\section{REFERENCES}

1. Kumar, V., A. K. Abbas, N. Fausto, and R. N. Mitchell. 2007 Robbins Basic Pathology, 8th ed. Saunders Elsevier.

2. Negi, S. S. 2005. Comparison of the conventional diagnostic modalities, bactecculture and polymerase chain reaction test for diagnosis of tuberculosis. Indian J. Med. Microbiol. 23:29-33.

3. World Health Organization, Bangladesh. 2004. Communicable diseases-Tuberculosis.

4. Mostofa, K., A. Jewel, and H. Shamim. 2009. Standard Operating Procedure (SOP) for Culture and DST of Mycobacteria, 1st ed National TB Control Program (NTP), Director General of Health Services (DGHS), Ministry of Health and Family Welfare, Bangladesh.

5. Bello, A. K., and C. H. Njoku. 2005. Tuberculosis: current trends in diagnosis and treatment. Niger. J. Clin. Pract. 8(2):118-124.

6. Githui W., S. M. Wilson, and F. A. Drobniewski. 1999. Specificity of IS6110-based DNA finger rinting and diagnostic techniques for Mycobacterium tuberculosis complex. J. Clin. Microbial. 37:12241226.

7. Uilukanligil, M., G. Aslan, and S. Tasci. 2000. A comparative study on the different staining methods and number of specimens for the detection of acid fast bacilli. Mem. Inst. Oswaldo. Cruz. 95(6):855858.

8. Bengisun, J. S., D. Karnak, I. Palabiyikoglu, and N. Saygun. 2000 Mycobacterium tuberculosis drug resistance in Turkey, 1976-97. Scand. J. Infect. Dis. 32:507-510

9. Salim, M. A. H. 2009. New hope in the treatment of MDR TB. Damien Foundation, Bangladesh.

10. Mahadev, B., et al. 2004. Surveillance of drug resistance to antituberculosis drugs in districts of hoogli in west bengal and mayurbhanj in orissa. Indian Journal of Tuberculosis. 48:129.

11. Iqbal, R., et al. 2008. Multidrug Resistance Tuberculosis in Lahore. Pak. J. Med. Res. 47:1 